\title{
La precarización salarial en la actividad agrícola y su incidencia en la política de economía popular y solidaria en el Ecuador
}

\author{
Luis Enrique Barreno Benavides, \\ Héctor Alejandro López Paredes, \\ Marco Vinicio López Paredes, \\ Gardenia Gonzales Franco*
}

\begin{abstract}
RESUMEN
La investigación presentada analiza cómo la precarización salarial agrícola incidió para la generación de emprendimientos productivos en la economía social y solidaria en Ecuador. La información recopila varios análisis de las principales causas que originan la precarización salarial agrícola, su posterior evaluación y la situación de la agricultura campesina, así como, un análisis de la evolución histórica de las empresas de economía social y solidaria. A la consulta bibliográfica, acompaña una investigación de campo aplicada a los actores de los emprendimientos productivos representados por organizaciones campesinas del sector de la economía social y solidaria , que se encuentran ubicados en la zona noroccidente de Pichincha (Ecuador), a quienes se aplicó una encuesta acompańada de varias reuniones para identificar las razones que motivaron su emprendimiento, permitiendo confirmar si los proyectos pertenecen a la política de economía social y solidaria. Los resultados permiten reconocer nuevas formas de emprendimiento a través de la asociatividad, así como, facilita información a las entidades gubernamentales para la aplicación de futuras políticas que incentiven el desarrollo de la economía social y solidaria.
\end{abstract}

Palabras clave: reforma agraria, precarización salarial, emprendimientos productivos, economía social y solidaria.

Clasificación JEL: A11, D2, E24

\footnotetext{
* Docente de la Universidad Tecnológica Equinoccial Quito - Ecuador lbarreno@ute.edu.ec, docente de la Universidad Tecnológica Equinoccial Quito - Ecuador hector.lopez@ute.edu.ec, docente principal investigador Pontificia Universidad Católica del Ecuador mvlopez@puce.edu.ec, Docente de la Universidad Tecnológica Equinoccial Quito - Ecuadorgfgv7063@ute.edu.ec
} 


\title{
Income insecurity in agricultural activity and its influence on the social and solidarity economy in Ecuador
}

\begin{abstract}
This study analyzes how agricultural income insecurity has affected the creation of productive enterprises in the social and solidarity economy of Ecuador. It draws on several studies of the main causes of agricultural income insecurity and their subsequent evaluation, the status of peasant agriculture, and the historical evolution of social and solidarity economy enterprises. It accompanies this bibliographical review with field research focused on actors involved in productive enterprises - represented by peasant organizations from the social and solidarity economy based in the northwestern part of Pichincha province (Ecuador) - through a survey and several meetings to identify the reasons behind their ventures and confirm whether they conform with the social and solidarity economy policy. The results allow recognition of new forms of entrepreneurship through associativity, as well as providing information to government entities for future policies that encourage the development of this sector of the economy.
\end{abstract}

Keywords: Agrarian reform, income insecurity, productive undertakings, social and solidarity economy.

JEL Classification: A11, D2, E24

\section{INTRODUCCIÓN}

La generación de emprendimientos productivos que se basan en los principios de Economía Social y Solidaria en el sector agrícola en el Ecuador, se analiza a partir de la revisión de la aplicación de la Ley de Reforma Agraria, que surge por las condiciones económicas de las familias campesinas, tomando como referencia la última reforma agraria que se dio en el sector rural de Ecuador en los ańos 1964 gracias la visión de Galo Plaza y Velasco Ibarra, se promulga en 1973 una nueva Ley de Reforma Agraria que requería la explotación eficiente de más del 80\% del predio; según Da Ros (2007) los ingresos de los campesinos que percibían como fruto de la venta de las actividades agrícolas y pecuarias disminuyeron considerablemente, al bajar los niveles de la productividad. Estos aspectos para Mills (2014) conllevan a aumentar los niveles de pobreza y, con ello, una reducción de los niveles de vida de los mismos, considerando que, a pesar de los esfuerzos de los distintos gobiernos para mejorar los niveles de producción a través de asesoramiento técnico y entrega de algunos insumos agrícolas como es de fertilizantes, abonos foliares y urea, entre otros, por cuanto, uno de los limitantes es el retaceo de las tierras, reduciéndose a lotes muy pequeños en los cuales es muy difícil sino imposible la aplicación de la tecnificación agrícola. Esto ha conllevado a que una gran cantidad de agricultores pequeños permanezcan con economías de sobrevivencia.

Viteri (2007) hace referencia a la necesidad tanto de familias campesinas dueños de los predios agrícolas pequeños, como aquellas que de una u otra forma trabajan en el 
sector agropecuario bajo un sistema remunerativo con ingresos precarios, se han visto en la necesidad de buscar otras alternativas en actividades productivas que permitan mejorar sus ingresos y con ello mejorar su situación económica y nivel de vida, encontrando en proyectos de emprendimiento productivos, ya sea en el ámbito artesanal aprovechando los recursos de la zona, de turismo comunitario o de la propia actividad agropecuaria, en el ámbito de la economía solidaria, la alternativa de solución a sus problemas.

En el caso de Ecuador, el estudio respondió a la pregunta «¡en qué medida la precarización salarial agrícola fue una de las causas en la formación de asociaciones de economía solidaria?», evidenciando que el factor principal que ha motivado emprendimientos agrícolas en la economía social y solidaria es la precarización salarial como consecuencia de las reformas agrarias que se han presentado en el país, con lo cual, los principales aportes del estudio servirán en primer lugar para reconocer a la economía social y solidaria como una alternativa de producción que respeta la participación comunitaria y solidaria en la generación de ingresos y mejora de las condiciones de vida de este sector, en segundo lugar los resultados constituyen un aporte a los organismos gubernamentales responsables de la generación y aplicación de políticas para incentivar la creación de emprendimientos productivos en el campo de la economía social y solidaria.

\section{ESTRUCTURA DE LA PROPIEDAD AGRÍCOLA}

Los proyectos de economía solidaria tienen un alto porcentaje de origen en el sector agropecuario en la mayoría de las personas que viven en el área rural del Ecuador, en donde, según lo expresado por Zeledón (s.f.) existen dos grandes etapas en la evolución y construcción de una ciencia para el derecho agrario: la primera comprendida en el período clásico entre 1922 y 1962, el cual se centró en su autonomía y especialización, siendo sus principales promotores Bolla y Arcangeli; y la segunda correspondiente al período moderno entre 1962 y 1998, teniendo como principal promotor a Antonio Carrozza, quien impulsa estudios de derecho comparado, logrando construir las bases de la nueva ciencia agrarista e incentivando la creación de organizaciones como la Unión Mundial de Agraristas Universitarios.

Según Viteri (2007) los sistemas de tenencia de la tierra en América Latina se han basado históricamente en la propiedad privada, esto es, la concentración de las tierras agrícolas en pocas familias y en la existencia de gran cantidad de familias campesinas sin tierra. De esta forma la reforma agraria en el Ecuador se origina como un mecanismo empleado para incluir al campesino ${ }^{1}$ en un contexto productivo, realizar una distribución adecuada de tierras (latifundios y minifundios) ${ }^{2}$ e incentivar la inclusión social.

\footnotetext{
1 Campesino según Chayanov se concibe en relación con una economía, una cultura o una clase social, de acuerdo a distintas tradiciones conceptuales, que en el contexto ecuatoriano se considera a la persona que proviene del campo o que se relaciona con él.

2 El Diccionario de la Real Académica de la Legua Española (RAE) define latifundio como «Finca rústica de gran extensión; y al minifundio como «Finca rústica que, por su reducida extensión, no puede ser objeto por sí misma de cultivo en condiciones remuneradoras»
} 
Agropecuarios (1964) señala que una reforma agraria integral se identifica con el desarrollo social y económico, porque de acuerdo a Zeledón (n.d.) persigue la incorporación de la población rural al desarrollo social y económico del país, mediante la justa y equitativa distribución de la tierra y la más alta eficiencia en su explotación, en una base de independencia y libertad para el hombre que vive del trabajo sobre la tierra.

Desde una evaluación cualitativa la Reforma Agraria fue un proceso social y político que juntó a los intereses de varias clases sociales, en primer lugar de las clases campesinas que luchaban contra el latifundio, con una perspectiva de cambio (como lo expresa la consigna: «tierra, trabajo y libertad»), luego los sectores intelectuales sensibilizados ante la magnitud de la explotación que sufrían los campesinos e indígenas, y también una fracción de la burguesía que presionaba por la terminación del latifundio tradicional y atrasado (Frank Brassel, 2008, p. 239).

Velasco (1979) hace referencia a la importancia de señalar que la corriente desarrollista representada en los gobiernos militares de aquel entonces, que llevaban la marca de la Alianza para el Progreso, cuyo lema principal fue la famosa revolución verde ${ }^{3}$ en los procesos productivos agrarios, no pudieron cumplir su legado principal de constituir una verdadera revolución agraria, siendo una de las razones por las que la reforma no fue más radical, la inexistencia de un movimiento social de carácter nacional que presionara uniformemente por una demanda más amplia que la del huasipungo. Otra razón fue la debilidad del modelo reformista en razón de que la reforma agraria en Ecuador tuvo sentidos distintos en la región andina y en la región costera; en la primera, era necesario redistribuir la propiedad agrícola, en la segunda no había esa necesidad, pues se trataba de una región en franca expansión de su frontera productiva, mediante la colonización.

Considerando lo expresado por Brassel, Herrera, y Laforge (2008) las reformas agrarias en el Ecuador tuvieron como base tres leyes: las de 1964, 1973 y 1979, siendo el punto crítico lo sucedido entre las décadas de 1960 y 1970 por la interpretación que se generó en ese entonces a los efectos económicos, sociales y políticos para la construcción de las reformas en el campo agrario, demostrando según Alain de Janvry (1991) que el modelo no era perjudicial solo para la agricultura, sino en modo particular para la producción de alimentos de consumo interno, en especial serranos, además excluyente en forma creciente de las economías campesinas.

El modelo agroexportador benefició a los grandes terratenientes ${ }^{4}$ de la región Costa, especializados en la producción de banano, café y cacao, en relación con su incremento de ingresos por la colocación de sus productos en mercados externos, en contraste, la oferta de productos agropecuarios provenientes de la sierra ecuatoriana destinados al consumo interno disminuyo afectando sus ingresos, así como, a los consumidores de

\footnotetext{
3 Denominación empleada para describir el incremento de la productividad agrícola y de alimentos entre 1960 y 1980 en Estados Unidos.

4 terrateniente: persona que es dueño de tierras o fincas rurales extensas.
} 
los centros urbanos. La reducción de la oferta de productos agrícolas y pecuarios ${ }^{5}$ para el mercado interno estuvo acompañada de la precarización salarial agrícola de aquellas personas que disponían únicamente de la venta de su fuerza laboral, debido a la baja productividad agropecuaria ${ }^{6}$ ocasionada por la aplicación de la reforma agraria impulsada por el gobierno de León Febres Cordero en 1984 que favoreció a los agroexportadores de la costa, lo que motivo en 1994 al gobierno de Sixto Durán Ballén a emitir la Ley de Desarrollo Agrario para contrarrestar el efecto negativo de esta reforma en los productores agrícolas de la sierra.

Una de las consecuencias directas de la Ley de Reforma Agraria se refleja en la tenencia de la tierra, por esta razón se analizará cuál es la situación de la misma en nuestro país según lo revisado por Brassel, Herrera y Laforge (2008) y Zeledón (2004), esto porque una de las razones para la formación de asociaciones de economía solidaria es precisamente la mala distribución de la tierra. En este sentido se afirma que el campo se constituyó, en una de las prioridades de las políticas de ajuste, promoviendo la privatización del territorio a través de la incorporación del mercado de tierras en zonas clave donde se concentra propiedades, infraestructura vial, energética y agua. Mientras que para las economías campesinas donde solo hay atención para aquellas integradas a los modelos de agronegocios ${ }^{7}$, vinculadas al mercado externo (Frank Brassel, 2008).

Daza Cevallos (2015), haciendo referencia a datos del III Censo Agropecuario de 2001, señala que el $94,53 \%$ de la tierra en el Ecuador se encuentra bajo un régimen de propiedad privada con cerca de 11680469 ha, superficie sobre la cual se extienden 828267 unidades productivas agropecuarias (UPA) de las cuales el 98,27\% se encuentran distribuidas entre grandes, pequeñas y medianas. Por su parte, la propiedad comunal sobre la tierra posee 602862 ha, con 13408 UPA y, 73261 ha, de las que 1228 UPA son propiedad del Estado. La estructura actual de la tenencia de la tierra en UPA según datos a 2016 extraídos del Instituto Nacional de Estadística y Censo (INEC) (Ver cuadro 1) muestran los siguientes resultados:

Cuadro 1. Estructura de las UPA en Ecuador

\begin{tabular}{lcccc}
\hline & UPA & PORCENTAJE & SUPERFICIE & PORCENTAJE \\
\hline \multicolumn{1}{c}{ TOTAL } & $\mathbf{8 4 2 8 8 2}$ & $\mathbf{1 0 0}$ & $\mathbf{1 2 3 5 5 8 3 1}$ & $\mathbf{1 0 0 , 0}$ \\
\hline Menos de 1 ha & 248398 & 29.5 & 95834 & 0,8 \\
De 1 hasta menos de 2 ha & 117660 & 14.0 & 156016 & 1,3 \\
De 2 hasta menos de 3 ha & 78850 & 9.4 & 183354 & 1,5 \\
De 3 hasta menos de 5 ha & 90401 & 10.7 & 339021 & 2,7 \\
\hline
\end{tabular}

Fuente: INEC 2016. Elaboración: Autores.

\footnotetext{
5 pecuario: del ganado o de la ganadería, o relacionado con ellos.

6 agropecuario: de la agricultura y la ganadería o relacionado con ellas.

7 agronegocio: actividades económicas asociadas con: producción de insumos y bienes de capital destinados a la producción agrícola. Producción agrícola.
} 


\begin{tabular}{lcccc}
\hline & UPA & PORCENTAJE & SUPERFICIE & PORCENTAJE \\
\hline \multicolumn{1}{c}{ TOTAL } & $\mathbf{8 4 2 8 8 2}$ & $\mathbf{1 0 0}$ & $\mathbf{1 2 3 5 5 8 3 1}$ & $\mathbf{1 0 0 , 0}$ \\
\hline De 5 hasta menos de 10 ha & 101066 & 12.0 & 688987 & 5,6 \\
De 10 hasta menos de 20 ha & 75660 & 9.0 & 1017807 & 8,2 \\
De 20 hasta menos de 50 ha & 76792 & 9.1 & 2372027 & 19,2 \\
De 50 hasta menos de 100 ha & 34498 & 4.1 & 2242409 & 18,1 \\
De 100 hasta menos de 200 ha & 12941 & 1.5 & 1666879 & 13,5 \\
De 200 hectáreas y más & 6616 & 0.8 & 3593496 & 29,1 \\
\hline
\end{tabular}

Fuente: INEC 2016. Elaboración: Autores.

Como se aprecia en el cuadro 1, el mayor porcentaje de unidades productivas agropecuarias está en las propiedades menores a una hectárea, alcanzando el 29,5\% del total; sin embargo, en superficie apenas representa no alcanza ni al 1\%. Si sumamos a ellos las UPA de uno hasta menos de dos hectáreas, la situación es aún más dramática, ya que alcanza a un $43,5 \%$, lo cual quiere decir que casi el 50\% de UPA tienen extensiones de tierras muy pequeñas. Estas cifras comprueban que un gran número de campesinos son dueños de pequeñas parcelas, en donde, difícilmente pueden aplicar algún tipo de tecnología agrícola para elevar o mejorar la productividad en sus unidades productivas y de esta forma mejorar sus paupérrimos ingresos caracterizándose más bien por mantener economías de subsistencia, fruto de ello es la presencia de altos niveles de pobreza en el área rural del país; este tipo de propiedades tiene mayor presencia en la región sierra.

Por otro lado, un reducido número de campesinos que pueden ser empresarios agrícolas representan el $0,8 \%$, de propiedades de 200 hectáreas o más, pero en extensión de tierra representa el $29,1 \%$ del total nacional, estando en capacidad de producir en economías de escala una variedad de productos con niveles aceptables de rendimiento, fruto de la aplicación de la tecnología agrícola que les permite obtener rendimientos productivos adecuados; estas características tienen mayor presencia en la región costa.

Esta polaridad en la propiedad de los predios rurales marca la inequidad en la zona rural del país (Sipae, 2011). Ecuador es uno de los países más pequeños de América del Sur; sin embargo, tiene uno de los índices más altos de inequidad en el acceso a la tierra. La superficie total del Ecuador es de $256370 \mathrm{~km}$ y el coeficiente de desigualdad en el acceso a la tierra (Gini sobre la tierra) es de 0,81: resulta que el 99,99\% de las UPA tienen extensiones inferiores a 640 ha (827 439 UPA) y solo el 0,001\% de las UPA tienen extensiones mayores a 640. Frente a esta realidad, la propuesta más reciente de democratizar la propiedad de la tierra o la redistribución de la misma, se genera en el gobierno de Rafael Correa. A pesar de ello, el 7 de enero de 2016 se aprobó la Ley de Tierras Rurales y Territorios Ancestrales, pero sin la afectación a los grandes latifundios, por cuanto solo califica como latifundios cuando la tierra en grandes extensiones no está correctamente aprovechada, lo cual ayudaría para evitar que grandes extensiones de tierras no se encuentren abandonadas. Además, se especifica que si la tierra no tiene el uso adecuado 
puede ser expropiada y declararse de utilidad pública; sin embargo, no se da una explicación sobre cuáles son las características de aprovechar correctamente las tierras.

En relación con la prohibición del latifundio, la Ley de Tierras y Usos Ancestrales manifiesta Ecuador (2016) que el Estado hará efectiva la prohibición del latifundio e impedirá la concentración de la tierra rural. Si bien la ley en mención señala la prohibición del latifundio, no da una definición clara de lo que es un latifundio, dejando en forma ambigua la interpretación de la misma. En este mismo ámbito, la ley especifica el concepto de unidad productiva familiar (UPF) Ecuador (2016) una unidad de medida económica estimada en un número de hectáreas de tierra productiva, que le permite a una familia rural percibir ingresos necesarios para satisfacer sus necesidades básicas que garantice el buen vivir, y que contribuya a la formación de un patrimonio.

Dentro de este ámbito, la cuestión es el rol de un modelo de desarrollo que respete y promueva a la agricultura campesina como una de sus estrategias para garantizar equidad, inclusión y desarrollo sustentable (Brassel, 2008).

\section{PRECARIZACIÓN SALARIAL EN EL ÁREA RURAL}

Para responder a la pregunta principal de la investigación, «¿En qué medida la precarización salarial agrícola fue una de las causas en la formación de asociaciones de economía solidaria?», se pasa al análisis de las situaciones que describe el concepto de precariedad ${ }^{8}$ : inseguridad en el empleo, bajos salarios, disminución de derechos laborales, dependencia personal respecto al empleador, malas condiciones de trabajo, ausencia de reconocimiento profesional (entre otros: Cano, 2000). Lo afirmado por Cano se refleja en las características que definen la pobreza en la zona rural, como los bajos niveles de ingresos que perciben las personas que venden su fuerza de trabajo en calidad de asalariados agrícolas, y que perciben salarios que no alcanzan para cubrir sus necesidades básicas, a lo que se suma la incertidumbre, seguridad social, falta de garantía entre otras. Además, las razones para que se presente la precarización laboral en el campo son varias; entre ellas la baja rentabilidad que obtienen los agricultores dueños de los medios de producción, el incumplimiento de la normatividad en el campo salarial en la zona rural por parte de los empleadores, el poco interés de las autoridades de control laboral y aseguramiento social entre otros, situación que se ve agrava con el capitalismo y su proceso de globalización.

Además de las causas señaladas de tipo general, hay otras específicas, como es el caso de no recibir el salario que le corresponde por ley, el hecho de no estar asegurado, inclusive por trabajar en ambientes insalubres, contaminados o muy fríos o calurosos. La situación para el trabajador agrícola y pecuario podría agravarse considerablemente al no tener estabilidad en el trabajo, no contar con un contrato; esto trae consecuencias lamentables, como el no poder planificar para adquirir los enseres domésticos básicos

8 precaridad: carencia o falta de estabilidad o seguridad. 
para el desarrollo normal de las actividades en la producción de alimentos y bienes básicos y peor aún una vivienda que le permita mantener un nivel digno de vida.

A continuación, se presenta algunos indicadores al año 2012 proporcionados por el INEC, que permiten confirmar lo manifestado. La información permite realizar un comparativo entre el área urbano y la rural hace las cifras de nuestro país que confirman lo señalado.

Cuadro 2. Comparativo de pobreza del área urbana y rural en Ecuador

\begin{tabular}{lrr}
\hline & \multicolumn{2}{c}{ ZONA } \\
\cline { 2 - 3 } \multicolumn{1}{c}{ INDICADOR } & URBANA & RURAL \\
\hline Porcentaje de viviendas con hacinamiento & $13,7 \%$ & $18,8 \%$ \\
Porcentaje de viviendas con abastecimiento de agua por red pública & $88,4 \%$ & $42,6 \%$ \\
Porcentaje de viviendas sin energía eléctrica & $0,3 \%$ & $5,1 \%$ \\
Porcentaje de viviendas sin alcantarillado & $27,3 \%$ & $82,4 \%$ \\
Porcentaje de hogares con teléfono convencional & $47,1 \%$ & $15,1 \%$ \\
Porcentaje de hogares con gasto de servicio de internet de la vivienda & $16,5 \%$ & $2,2 \%$ \\
Ingreso promedio del hogar & 1,046 & 567 \\
Ingreso corriente monetario del hogar & 841 & 208 \\
Ingreso por trabajo & 759 & 371 \\
Ingreso del trabajo dependiente (asalariado) & 702 & 386 \\
Ingreso del trabajo independiente (agropecuario y no agropecuario) & 426 & 164 \\
Ingreso corriente no monetario del hogar & 208 & 140 \\
Ingreso promedio per cápita de los asalariados (agricultores y trabajadores calificados) & 268 & 201 \\
Ingreso per cápita en general & 274 & 141 \\
Gasto corriente promedio del hogar & 943 & 526 \\
Gasto corriente per cápita & 247 & 131 \\
Porcentaje del gasto en alimentación & $22,6 \%$ & $32,0 \%$ \\
Porcentaje de población asegurada & $38,8 \%$ & $31,2 \%$ \\
Escolaridad promedio de personas de 24 años y más & 10,6 & 6,5 \\
Porcentaje de personas de 15 a 17 ańos que asisten a educación media & $64,0 \%$ & $49,1 \%$ \\
Menos de 1 SUB & 362050 & 489221 \\
De 20 SBU y más & 12223 & 798 \\
\hline
\end{tabular}

Fuente: INEC 2016. Elaboración: Autores.

Como se puede observar en el cuadro, las diferencias que existen en los indicadores referentes a nivel de ingresos, de gastos y en los demás indicadores, que de una u otra forma permiten calificar el nivel de vida de las familias comparadas entre el área urbana y rural, las diferencias son muy grandes, siendo siempre desfavorable al del área rural. Todo esto no hace más que ratificar lo que en este documento se ha manifestado, es decir 
que la gran mayoría de la población del área rural vive en condiciones de vida deplorables debido a los escasos recursos que percibe en sus actividades productivas. Esta es una de las causas para que muchos de ellos traten de emprender en emprendimientos productivos en el marco de la Economía Solidaria, es decir en pequeños proyectos en donde trabaja toda la familia en el proceso de producción y venta de bienes y servicios caracterizados por pequeñas inversiones en activos fijos tangibles, en procesos de producción con predominio de los recursos humanos al de capital.

\section{ECONOMÍA POPULAR Y SOLIDARIA: SECTOR REAL}

En razón que los proyectos en Economía Solidaria tienen como base los emprendimientos productivos, es necesario realizar un pequeño análisis de lo que significa el término emprender. Según Alcaraz Rodríguez (2011) «emprender» es un término que tiene múltiples acepciones, según el contexto que se lo emplee, será la connotación que se lo adjudique. En el ámbito de los negocios, el emprendedor es un empresario, es el propietario de una empresa comercial con fines de lucro. Las características de un buen emprendedor son: compromiso total, capacidad para alcanzar metas, orientación a las metas y oportunidades, iniciativa y responsabilidad, persistencia en la solución de problemas, realismo, autoconfianza, altos niveles de energía, busca de realimentación, control interno alto, toma de riesgos calculados, baja necesidad de estatus y poder, integridad y confiabilidad y tolerancia al cambio, Alcaraz Rodríguez (2011).

Al hablar de economía popular y solidaria el concepto de emprendimiento de acuerdo a lo manifestado por Osorio (2016) se entiende como las relaciones horizontales que conforman un grupo heterogéneo de apoyo mutuo en respeto del medio ambiente, sostenibilidad de la vida, basando su relación en los principios de solidaridad, equidad, autonomía, comunidad y permacultura para gestionar la vida en colectivo, esto significa que el emprendimiento constituye una forma de producción para la sobrevivencia que no distingue clases sociales, autoridad ni apropiación individual, pues el resultado de la producción es repartido en forma solidaria e igualitaria entre los miembros de la comunidad.

Todas las características señaladas se combinan en forma adecuada con lo que les gusta hacer, con el conocimiento que posea. Muy importante es también aprovechar de las habilidades que se disponga, combinado con alguna experiencia en la producción de los bienes y los servicios y siempre pensando en lo que se quiere alcanzar en el futuro.

La economía solidaria, fecha el comienzo del cooperativismo moderno en 1844 con la creación de la «Rochdale Society of Equitable Pioneers», cuyos principios siguen siendo la base a partir de la cual se define hoy una cooperativa. En realidad, esta cooperativa ni

9 Rochdale es una ciudad situada en el Gran Mánchester (noroeste de Inglaterra) y atravesada por el río Roch. La ciudad es conocida por ser el lugar de nacimiento del movimiento cooperativo. 
siquiera fue la primera cooperativa de consumo británica: la «Sociedad de las Hilanderas de Fenwick» había sido creada ya en 1769. La importancia que se le ha otorgado posteriormente se debe a que se trató de un temprano "caso de éxito» de una cooperativa de consumo. Rochdale era un buen «mito fundacional» para un movimiento que pretendía marcar distancias con los movimientos revolucionarios de Europa continental y su insistencia en que el centro del «problema social» estaba en la propiedad de los medios de producción. Hasta hace tan solo un par de años, en el anglo mundo todavía se definía cooperativa como una empresa propiedad de sus consumidores. El cooperativismo toma como base la libertad/mutualista de Fourier, la práctica socialista de kibutz y la cristiana del padre José María Arizmendiarrieta, que se caracterizaron por la producción, la relación con el Estado y la intervención de la iglesia en la producción bajo una corriente asistencialista ${ }^{10}$, tomando como base principios de libertad, igualdad, la participación y la solidaridad.

En Europa el desarrollo de la cooperación mantuvo varias dificultades y se generó en tiempos muy concentrados de poder, dando forma a las organizaciones actuales como son cooperativas de producción, ya sea en el ámbito agrícola, o industrial, en cooperativas de consumo y las cooperativas de ahorro y crédito y algunas sociales que nacieron en Italia entre las décadas de 1980 y 1990 en respuesta a una falta del Estado en brindar ciertos servicios de naturaleza social en términos de welfare state $^{11}$.

En la década de 1970, la economía social experimenta un incremento muy importante, en especial en el continente europeo y en algunos países sudamericanos como es el caso de Argentina, Ecuador, Chile y Brasil, en parte por el debilitamiento de los Estados en la generación y sostenimiento del mismo, lo que llevó a ideas para generar autoempleo y acciones que permitan acercar a las cooperativas al mercado y acceder a varios servicios sociales existentes entre los actores económicos y, a su vez, disminuir la pobreza de aquellos sectores poblacionales con escasos recursos económicos. De esta definición se desprenden dos figuras: la una con un importante aporte de tipo socioeconómico, en donde se analiza el riesgo económico para las inversiones y una marcada autonomía con la presencia de un trabajo asalariado que beneficia a sus socios o integrantes; $y$, por otra parte, una de tipo social, que se centra en beneficiar a la comunidad o a un grupo específico de personas, no tiene como principal prioridad el reparto del capital y más bien una distribución limitada de beneficios.

En el Ecuador, el origen y consolidación del cooperativismo, citado por Da Ros en el artículo "El movimiento cooperativo en el Ecuador. Visión histórica, situación actual y perspectivas» en 2007 , se da en tres etapas fundamentales: a) la primera se

\footnotetext{
10 Asistencialismo es una forma de aplicación de la asistencia, obligación que contraen los gobiernos con sus ciudadanos a través de una constitución, por la cual se señala el carácter de dignidad de todo ser humano sin distinciones de ningún tipo.

11 Welfare state es un concepto de gobierno en el cual el Estado desempeña un papel clave en la protección y promoción del bienestar social y económico de sus ciudadanos.
} 
inicia aproximadamente en la última década del siglo XIX, cuando se crean especialmente en Quito y Guayaquil una serie de organizaciones artesanales y de ayuda mutua; b) la segunda empieza a partir de 1937, año en el cual se dicta la primera Ley de Cooperativas con el propósito de dar mayor alcance organizativo a los movimientos campesinos, modernizando su estructura productiva y administrativa, mediante la utilización del modelo cooperativista: c) la tercera etapa comienza a mediados de la década de 1960 con la expedición de la Ley de Reforma Agraria (1964) y de la nueva Ley de Cooperativas (1966) (Da Ros, 2007).

Si bien el objetivo de la creación del sistema cooperativo era el de corregir las desigualdades socioeconómicos que predominaba en el sector rural y en especial en el agro, el movimiento cooperativo con mayor fuerza se desarrolló en las dos grandes ciudades ejes del crecimiento económico del país, Quito y Guayaquil, mientras que en el área rural solo eran pequeńas e incipientes organizaciones especialmente campesinas que venían trabajando en pequeños emprendimientos artesanales sin mayores técnicas productivas y peor aún organizacionales en pos de conseguir mejores ingresos para sus familias, sus productos los comercializaban generalmente en los mercados domésticos más cercanos a sus domicilios.

Según lo expresado por Arcos y Palomeque (1997) a partir de los años ochenta, se incorporan nuevas temáticas que provocan cambios sustanciales en el contexto de la cooperación internacional y a nivel del papel del Estado en la economía y sociedad, en donde, para Cajamar (2004) a las empresas que están dentro del sector del no mercado se las debe considerar parte de la economía social, denominándolas de diferentes formas como: del tercer sector, sector excepto de impuestos, sector filantrópico, voluntario y caritativo, sector no lucrativo, sector intermedio, sector independiente y subsector de la economía social:

En el desarrollo histórico, en la búsqueda de alternativas económicas al capitalismo, las diversas experiencias de la economía social y solidaria demuestran una serie de potencias o capacidades transformadoras, que hacen valioso al sector para el cambio social, en la medida que se orienta hacia la satisfacción de necesidades humanas y no solo hacia la maximización de beneficios, haciéndolo de forma democrática y sostenible (Guridi, 2012).

La diferencia de una empresa capitalista de una de economía social, se debe buscar en el comportamiento de la atribución de sus resultados generados y en el proceso de toma de decisiones, se señalan algunas formas de hacerlo. La concepción organicista, considera que una empresa corresponde a una de economía social cuando su actividad productiva se basa en técnicas de organización fundamentadas en principios de organización y participación; si bien la economía de mercado constituye la esencia de la economía social, sin embargo, el éxito está en delimitar correctamente el campo de la economía social, de hecho en esta última necesariamente debe existir un beneficio para que tenga razón su existencia, pero los beneficios generados por la empresa no deben estar ligados 
directamente con los dueños del capital, de igual forma los votos para las decisiones en las de economía social deben ser un hombre un voto a diferencia de las del mundo capitalista que los votos corresponde al peso de las acciones o participaciones del capital, sin embargo de todo esto, no quiere decir que las economía social no sea eficientes, todo lo contrario de demostrar un alto nivel de eficiencia en sus actividades productivas para que la empresa se desarrolle y progrese considerando el nuevo desafío del entorno económico mundial la globalización y la competitividad.

Por lo antes señalado y en razón a que no existe un concepto único y específico sobre economía solidaria sino que se enmarca dentro de la definición de economía social, se pasa a mencionar la definición de economía social, término que se ha venido popularizando en la literatura económica para definir aquellas empresas y organizaciones privadas que, fundamentadas en los principios de la solidaridad y ayuda a sus asociados, se rigen por prácticas de democracia económica, dando primacía a las personas frente al capital (Cajamar, 2004) En el Ecuador a partir de 2011; año en el cual se emite la Ley Orgánica de Economía Popular y Solidaria y del Sector Financiero y Solidario (LOEPS), en donde se define a la EPS (Solidaria, 2014) como una forma de organización económica en la que sus integrantes, ya sea individual o colectivamente, organizan y desarrollan procesos de producción, intercambio, comercialización, financiamiento de bienes y servicios, mediante relaciones basadas en la solidaridad, cooperación y reciprocidad, situando al ser humano como sujeto y fin de su actividad, es decir, el trabajo sobre el capital.

La ley establece en forma clara los siguientes principios para quienes realicen actividades productivas basadas en esta:

a) La búsqueda del buen vivir y del bien común;

b) La prelación del trabajo sobre el capital y de los intereses colectivos sobre los individuales;

c) El comercio justo y consumo ético y responsable:

d) La equidad de género;

e) El respeto a la identidad cultural;

f) La autogestión;

g) La responsabilidad social y ambiental, la solidaridad y rendición de cuentas; y,

h) La distribución equitativa y solidaria de excedentes.

Con esta ley el Estado ecuatoriano busca colocar dentro de este marco jurídico a todas las personas, individuales y colectivas, naturales y jurídicas, que emprendan en actividades productivas de bienes y servicios que cumplan con las características expuestas en el instrumento legal señalado, para que de esta forma se pueda exigir y cumplir las obligaciones previstas en la misma. En el Ecuador la definición involucra a la palabra popular y solidaria, además esta definición no hace relación sobre la forma de la retribución del capital a través del beneficio generado, lo que deja abierto para que cada 
empresa u organización lo decida en función de sus propios intereses muchas veces olvidándose del carácter solidario y comunitario

Giovanni Cardoso, director nacional de la Corporación de Finanzas Populares y Solidarias, durante su ponencia en la Universidad Tecnológica Equinoccial en la Semana Internacional de la Economía Social y Solidaria realizada en el mes de julio de 2016, señaló que en el sector de la agricultura, ganadería, caza y silvicultura se destinan el $42 \%$ de los recursos económicos para impulsar esta actividad, de los cuales el 53,4\% se colocan en sectores rurales y el 46,6\% en áreas urbanas, representando un total de 822804 732 dólares distribuidos de la siguiente manera:

Cuadro 3. Distribución de los préstamos por provincia colocados por la corporación nacional de finanzas populares y solidarias

\begin{tabular}{lcc}
\hline \multicolumn{1}{c}{ Provincia } & Monto USD & \% de la colocación \\
\hline Azuay & 60797137 & $7,4 \%$ \\
Bolívar & 38276191 & $4,7 \%$ \\
Cañar & 8388064 & $1,0 \%$ \\
Carchi & 25730024 & $3,1 \%$ \\
Chimborazo & 61651712 & $7,5 \%$ \\
Cotopaxi & 75835575 & $9,2 \%$ \\
El Oro & 22157337 & $2,7 \%$ \\
Esmeraldas & 9616769 & $1,2 \%$ \\
Guayas & 48448306 & $5,9 \%$ \\
Imbabura & 59228448 & $7,2 \%$ \\
Loja & 29589361 & $3,6 \%$ \\
Los Ríos & 47531338 & $5,8 \%$ \\
Manabí & 66491579 & $8,1 \%$ \\
Morona Santiago & 8239679 & $1,0 \%$ \\
Napo & 9138790 & $1,1 \%$ \\
Pichincha & 83597771 & $10,2 \%$ \\
Santo Domingo de los Tsáchilas & 32718685 & $4,0 \%$ \\
Tungurahua & 94591913 & $11,5 \%$ \\
Zamora Chinchipe & 17906696 & $2,2 \%$ \\
Demás provincias & 22869357 & $2,8 \%$ \\
\hline Total & 822804732 & $100,0 \%$ \\
\hline Fin Cas & & \\
\hline
\end{tabular}

Fuente: Cardoso Geovanny, ponencia congreso de economía solidaria Universidad Tecnológica Equinoccial, junio 2016. 


\section{ANÁLISIS EMPÍRICO}

En función de la pregunta central de investigación, «¿En qué medida la precarización salarial agrícola fue una de las causas en la formación de asociaciones de economía popular y solidaria?», la propuesta metodológica fue de tipo exploratoria, descriptiva y analítica; para la sustentación y desarrollo del marco teórico se tomó información secundaria de tipo documental, expuesta por distintos autores sobre el tema, permitiendo de esta forma conocer el criterio de ellos sobre el origen, su evolución y desarrollo hasta el momento actual de dichas organizaciones en el mundo y en especial en Ecuador. Para la recolección de datos se utilizó fuentes primarias a través de la investigación de campo utilizando como instrumento la encuesta, lo que permitió demostrar la pregunta central de investigación.

La población objetivo fue definida en base a personas que actualmente son miembros activos de organizaciones que tienen como finalidad el trabajo en emprendimientos productivos con las características de economía popular y solidaria, según la legislación ecuatoriana, determinando como zona geográfica el área de influencia de la Universidad Tecnológica Equinoccial, correspondiendo a aquellas que tienen su jurisdicción en los diferentes cantones de la provincia de Pichincha, tales como Puerto Quito, Pedro Vicente Maldonado, San Miguel de los Bancos, Pedro Moncayo, Cayambe, Mejía y Quito; la información estadística que se tomó para la determinación del universo fue la disponible en el Catastro de Organizaciones del Sector no Financiero proporcionada por la Superintendencia de Economía Popular y Solidaria del Ecuador actualizada a mayo de 2016, tomando en cuenta únicamente las consideradas como Organizaciones de Economía Popular y Solidaria de acuerdo a nuestra legislación.

Para determinar el tamaño óptimo de la muestra se aplicó el criterio de muestra finita, para lo cual se trabajó con una $(Z)$ o grados de confianza del $95 \%$ un nivel de error aceptable de un $5 \%$ y un $50 \%$ de aceptación y rechazo obteniendo un total de 100 miembros de un total de 56 asociaciones que cumplían las características de emprendimientos de economía social y solidaria definidas en la legislación ecuatoriana. El proceso partió por realizar el contacto con el representante de la organización, en la que se obtenía una cita señalándole el motivo de la misma para que el representante asigne a la persona más adecuada para dicho fin. La aplicación de la encuesta fue realizada directamente por los responsables de la investigación. El procesamiento de la información de campo fue a través del software estadístico del SPSS, el cual permitió medir en primera instancia la confiabilidad de las encuestas y luego a través de cruce de variables obtener los resultados que permitieron obtener las conclusiones y responder a la pregunta principal de la investigación.

La encuesta se aplicó a una muestra simple de 100 miembros de 56 asociaciones diferentes que trabajan en proyectos de Economía Popular y Solidaria en la sierra central del territorio ecuatoriano; esto se da en las provincias de Pichincha, Cotopaxi, Imbabura, 
Tungurahua y Chimborazo. La información para la aplicación de las encuestas y entrevistas se tomó del listado del Catastro entregado por la Superintendencia de Economía Popular y Solidaria de las Asociaciones no financieras del Ecuador, lo que permitió conocer el número de miembros de las cooperativas, las actividades o emprendimiento que desarrollan, las actividades anteriores a que se dedicaban antes de formar parte de la asociación actual, los productos que obtienen, las razones por las que decidió emprender, saber si ha mejorado su nivel de vida como producto del emprendimiento, la capacitación que han recibido y en cuanto esto le ha permitido mejorar su actividad dentro de la organización, si requiere capacitación adicional y en qué áreas del conocimiento lo requiere; dichos resultados se presentan a continuación:

Gráfico 1. Número de socios por asociación. Información recabada de la encuesta aplicada para la investigación y elaborada y procesada por los autores

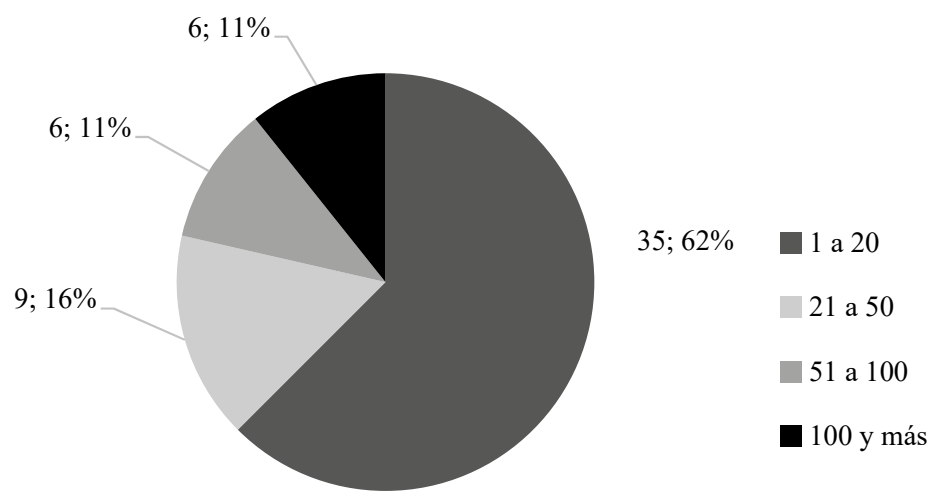

Fuente: Información recabada de la encuesta aplicada para la investigación (2017) y elaborada y procesada por los autores.

Para la muestra de las 56 asociaciones, si bien los resultados muestran una gran heterogeneidad en cuanto al número de socios por asociación, ya que presentan una amplitud de rango muy grande, la cual va desde 1 hasta 1384 socios. Analizando por rangos específicos, en función del rango de socios, se tiene que de 1 a 20 socios representan el $35 \%$, entre 21 y 50 representan el $9 \%$, entre 51 y 100 corresponde al $6 \%$ y con el mismo porcentaje de 100 en adelante, lo cual confirma que la muestra tomada es heterogénea. Sin duda alguna las asociaciones con el mayor número de socios son la Asociación Regional de soberanía Alimentaria del Territorio, RESAK, con 1384 socios, en segundo lugar, de la Asociación Banco Comunitario Atucucho con 400 socios y, en tercer lugar, Asoceralif con 153 socios. 
Gráfico 2. Tipo de actividad de las asociaciones

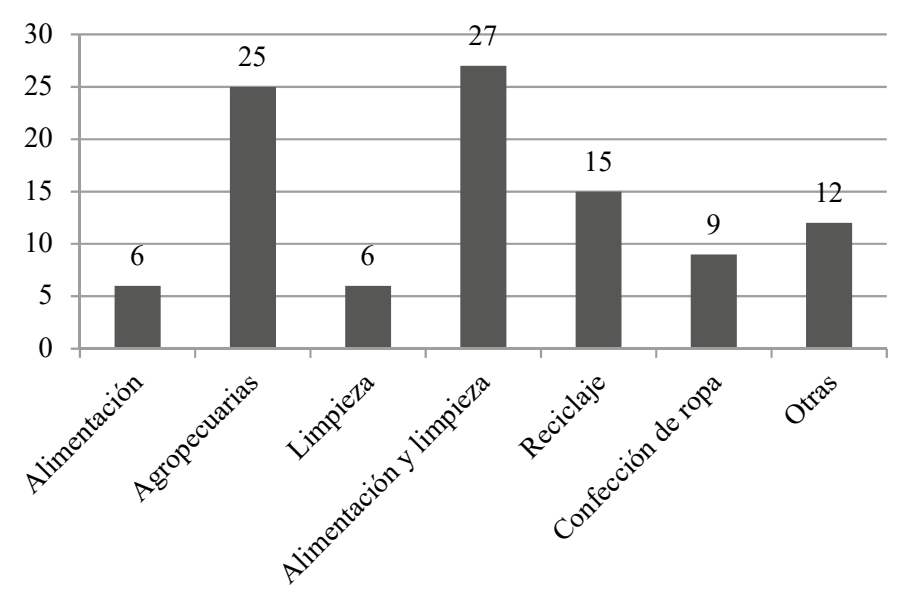

Fuente: Información recabada de la encuesta aplicada para la investigación (2017) y elaborada y procesada por los autores.

En lo referente a las actividades se aprecia un total de 32 diferentes actividades. Entre las más importantes están: con el $27 \%$ las que desarrollan servicios de alimentación y limpieza, seguido de un $25 \%$ de actividades agropecuarias, luego con el $15 \%$ dedicadas a reciclaje y venta de desechos sólidos y de un $9 \%$ confección de ropa, con el $6 \%$ están actividades por separado de alimentación y limpieza respectivamente y, el restante $12 \%$, son diversas actividades con pequeños porcentajes cada una.

Gráfico 3. Actividades de los socios antes de pertenecer a la asociación

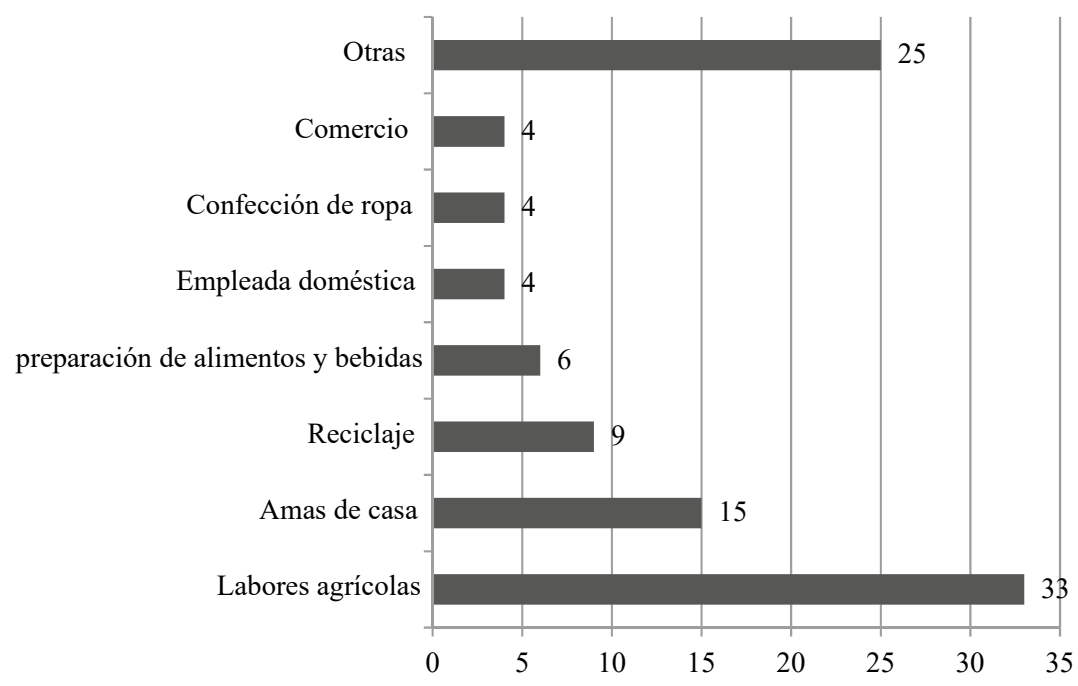

Fuente: Información recabada de la encuesta aplicada para la investigación (2017) y elaborada y procesada por los autores. 
Al preguntar a los estadísticos sobre cuáles habían sido sus actividades anteriores al trabajo en el emprendimiento, las respuestas viene a corroborar lo que se manifestó al inicio de la investigación, de que la incidencia para los emprendimientos productivos en su mayoría tenían relación con la precarización agrícola, por cuanto de las 100 personas miembros de las asociaciones consultadas, $33 \%$ de ellas realizaban anteriormente laboras agrícolas, al que le siguen las labores de ama de casa con el $15 \%$ y en tercer lugar está las labores de reciclaje con un 9\%. Como las encuestas fueron aplicadas tanto en el área urbana como rural, las distintas actividades que no corresponden a las agrícolas son las que realizan en el área urbana luego de haber inmigrado de las zonas rurales a las urbanas y realizadas especialmente por mujeres.

Gráfico 4. Formación académica de los integrantes de las asociaciones.

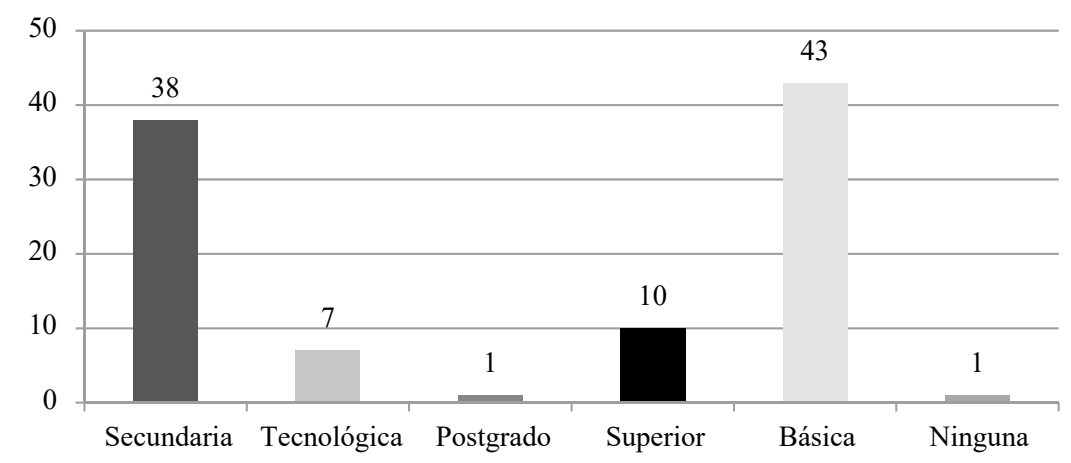

Fuente: Información recabada de la encuesta aplicada para la investigación (2017) y elaborada y procesada por los autores.

En la formación académica los miembros de las asociaciones consultadas indican que el 43\% tiene formación básica, un 38\% tienen formación secundaria y un 10\% superior.

Gráfico 5. Causas que generaron el emprendimiento en la asociación

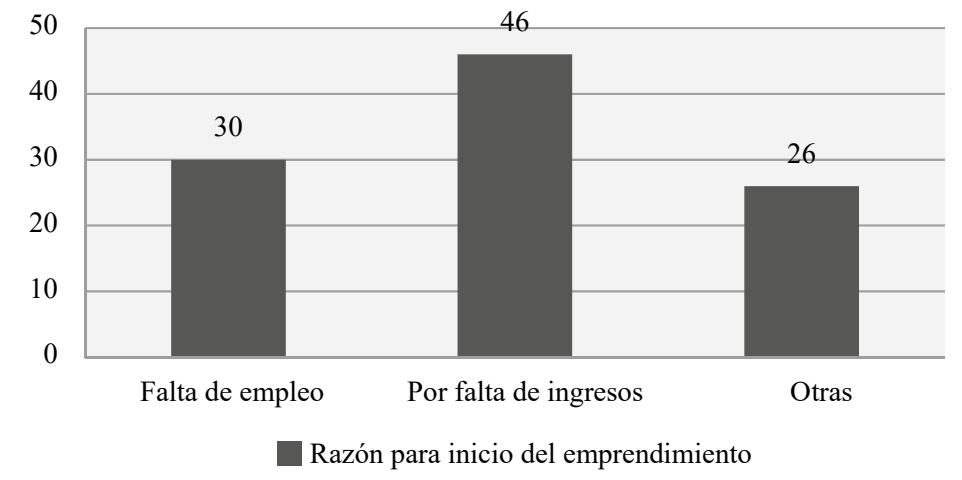

Fuente: Información recabada de la encuesta aplicada para la investigación (2017) y elaborada y procesada por los autores. 
Entre las razones más importantes que les motivó asociarse para iniciar esta actividad están las relacionadas con la falta de recursos o ingresos económicos de las familias, por un suman el $46 \%$, mientras que las relacionadas con la falta de empleo en sus diferentes expresiones suman el $30 \%$ y el restante $26 \%$ corresponde a otras razones.

Gráfico 6. Nivel de vida de los socios

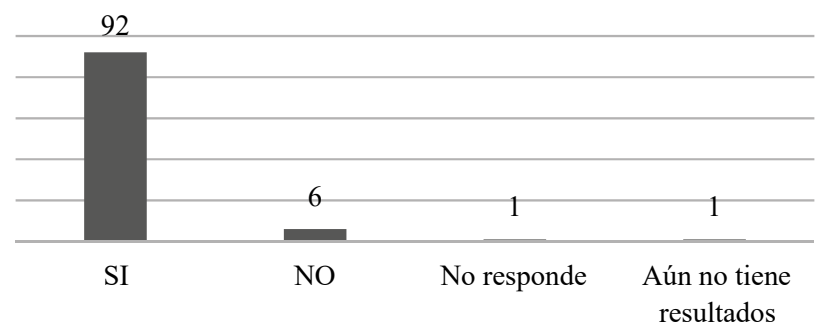

Ha mejorado su nivel de vida con los ingresos percibidos por su nuevo emprendimiento?

Fuente: Información recabada de la encuesta aplicada para la investigación (2017) y elaborada y procesada por los autores.

De los estadísticos obtenidos el $92 \%$ de los asociados consultados respondieron que sí ha mejorado el nivel de vida de ellos y su familia desde que iniciaron el emprendimiento asociativo, un $6 \%$ manifestó que no, y el 1\% manifestó que aún no tiene resultados.

\section{CONCLUSIONES Y DISCUSIÓN}

Los resultados de la investigación muestra que la mayoría de los emprendimientos productivos en el campo de la economía popular y solidaria provienen del sector rural o tienen su origen en el mismo, como lo demuestran el 33\% de los socios consultados al manifestar que sus actividades anteriores eran en laborares agrícolas antes de iniciar el emprendimiento actual, que a pesar de mantener la misma actividad se ha enfocado a través de la generación de una asociación de economía social y solidaria. Demostrando que estos emprendimientos tienen como una características común la condición de precarización salarial agrícola que motivó la asociación de personas de similares características para buscar una alternativa de producción y mejorar su condición de vida.

La causa principal para iniciar emprendimientos de Economía Popular y Solidaria está reflejada en un $46 \%$ de falta de ingresos de la población y en un $30 \%$ que no tienen empleo, evidenciando la limitación de recursos económicos obtenidos de la agricultura y ganadería, en donde, la producción agrícola y pecuaria es únicamente para subsistencia, siendo casi nula los excedentes de producción que no consumen las familias para destinarlos a la venta en mercados locales. Esta situación provoca un margen muy reducido de ingresos para los demás gastos del hogar, reflejando su alto nivel de pobreza 
y necesidades insatisfechas, siendo la razón que motiva a la gran mayoría de personas a asociarse para emprender en actividades que les genere algún ingreso adicional, ya sea en emprendimientos agrícolas y/o pecuarios.

Los emprendimientos que realizan en las asociaciones son muy diversos, pero las que más se destacan, especialmente en el área urbana son actividades de alimentación y limpieza con un $27 \%$, seguida de actividades agropecuarias con el $25 \%$. Hay que destacar que en estos últimos años se han visto incentivados por la demanda de alimentos preparados para las escuelas públicas, en la cual trabajan muchas asociaciones siendo proveedores del Estado a través del Servicio Nacional de Contratación Pública del Ecuador (Sercop), de igual manera las que trabajan en el sector textil y en especial en la confección de ropa, quienes proveen los uniformes escolares para escuelas públicas. Otros de los emprendimientos importantes están en el sector rural en proyectos agrícolas y pecuarios. En los primeros, en especial en la producción de productos agrícolas ecológicos u orgánicos, los que a más de dotar de importantes ingresos a sus asociados ofrecen alimentos sanos a la población.

Se señala que, a pesar de ser calificadas todas las asociaciones por el Instituto Ecuatoriano de Economía Popular y Solidaria (IEPS), lo cual quiere decir que todas ellas deben cumplir con el requisito básico de estar dentro de lo establece el marco legal para que se considere como una asociación de Economía Popular y Solidaria, sin embargo no todas lo cumplen, especialmente en la repartición del excedente generado por la actividad y la obligatoriedad de realizar obras de tipo social para que se beneficie toda población; algunas tampoco cumplen con el número de socios. Es de señalar también que muchas asociaciones no tienen un asesoramiento adecuado en cuanto a la estructura organizacional de las mismas y no tienen definidas un organigrama estructural ni funcional adecuado.

En lo referente a la ayuda que los miembros de las asociaciones reciben en capacitación, se puede establecer que la mayoría de las asociaciones consultadas han recibido importante capacitación, en especial de entidades del sector público, tales como: los Consejos Provinciales, el Instituto Ecuatoriano de Economía Popular y Solidaria (IEPS), la Agencia de Regulación, Control y Vigilancia Sanitaria (ARCSA), el Servicio Ecuatoriano de Capacitación Profesional (SECAP), los municipios y centros de educación como son las universidades públicas y privadas, además de organismos no gubernamentales ONG. Sin embargo, los miembros de las organizaciones deben autogestionar capacitación adicional relacionada con procesos de producción, contabilidad y finanzas, marketing, promoción y ventas, entre otros.

Finalmente se pudo constatar que, a pesar de que estas organizaciones están conformados por personas que supuestamente tienen con fin común el trabajo y cooperación solidaria en el que debe primar la honestidad, la ética y honradez de todos y cada uno de ellos y mucho más de sus dirigentes, sin embargo, en una visita a una organización del noroccidente de Pichincha, se pudo constatar la denuncia que en esos momentos 
estaban realizando los asociados al gerente de la misma por falta de informes y por sospechas de haberlos defraudado, solicitando inclusive una auditoria a la administración anterior, y a su vez se quejaban de la falta de intervención de los organismos encargados de realizar el control como es la Superintendencia de Economía Popular y Solidaria a pesar de las constantes solicitudes realizadas a dicha institución.

\section{REFERENCIAS BIBLIOGRÁFICAS}

$A C I$ (s.f.). Recuperado de ACI: http://www.aciamericas.coop/Principios-y-Valores-Cooperativos4456

Agropecuarios, I. I. (1964). Seminario sobre Programación y Administración de Reforma Agraria. Maracay: IICA.

Alcaraz Rodríguez, Rafael (2011). El emprendedor de éxito. México: Mc Graw Hill.

Arcos, Carlos y Edison Palomeque (1997). El mito al debate: las ONGs en Ecuador. Quito: AbyaYala.

Brassel, Frank, Stalin Herrera y Michel Laforge (agosto de 2008). Flacsoandes. Recuperado de http://www.flacsoandes.edu.ec/libros/digital/41947.pdf

Brassel, Frank, Stalin Herrera y Michel Laforge (2008). Reforma Agraria en el Ecuador, Viejos temas, nuevos argumentos. Quito: AVSF, Intermón Oxfam, IRD.

Cajamar, Instituto (2004). Economía social. La actividad económica al servicio de las personas. Juan Francisco Juliá Igual, coord.). Almería: Caja Rural Intermediterránea.

Cano, Ernest (2000). Análisis de los procesos sociales de precarización laboral. Precarización laboral,flexibilidad y desarrollo.

Cardoso, Geovanny (2016). Finanzas populares solidarias entre 2007 y 2016. Semana de la Economía Social y Solidaria UTE. Quito.

Chaves, Rafael (2008). La Economía Social: dos décadas generando empleo, tegido productivo y cohesión social en Europa. Valencia: Instituto Universitario de Economía Social y Cooperativa de la Universidad de Valencia.

Da Ros, Giuseppina (2007). El movimiento cooperativo en el Ecuador. Visión histórica, situación actual y perspectivas. Recuperado de://C:/Users/YOGA/Desktop/Andreina utpl/tesis/ bibilografia/pdf/El-movimiento-cooperativo-Ecuador.pdf

Daza Cevallos, Esteban (20 de junio de 2015). lalineadefuego. Recuperado de https:// lalineadefuego.info/2015/06/23/problematicas-de-la-tierra-en-el-ecuador-por-esteban-daza-cevallos/

de Janvry, Alain (1991). Estrategias para mitigar la pobreza rural en America Latina y el Caribe: Encadenamientos de producción en la economía campesina en el Ecuador. San José: Fondo Internacional de Desarrollo Agrícola- ICCA.

Defourny, Jacques y Marthe Nyssens (2012). El enfoque EMES de empresa social desde una perspectiva comparada. CIRIEC - España Revista de Economía Pública Social y Cooperativa, 75, 7-34.

Ecuador, Asamblea Nacional (07 de marzo de 2016). Ley Orgánica de Tierras Rurales y Territorios Ancestrales. Ley Orgánica de Tierras Rurales y Territorios Ancestrales. Quito.

Guridi, Luis, Juan Carlos Pérez de Mendiguren Castresana, Ana Iametti Señoriño, María Victoria Deux Marzi, Gonzalo Vázquez y Amaia Uribe (2012). Experiencias de Economía social y Solidaria: Compartiendo Estrategías y Aprendizajes. Papeles de Economía Solidaria. 
Osorio, María Daniela (2016). Economía(s) solidaria(s) y sostenibilidad de. Economía Crítica, 178-198.

SIPAE (2011). Tenencia de la tierra en Ecuador. Quito: Impresos Miraflores.

Solidaria, S. D. (2014). Ley Orgánica de Economía Polular y Solidaria. Registro Oficial. Quito, Pichincha, Ecuador: Intendencia de Estadísticas, Estudios y Normas de la EPS y SFPS .

Velasco, Fernando (1979). Reforma agraria y movimiento campesino indígena de la sierra. Quito: Editorial el Conejo.

Viteri Díaz, Galo (2007). eumed. Recuperado de Reforma Agraria en el Ecuador. http://www. eumed.net/libros-gratis/2007b/298/ley-1973.htm

Zeledón, Ricardo (s.f.). abda.com. Recuperado de http://www.abda.com.br/texto/zeledonzeledon.pdf

Zeledón, Ricardo (2004). Estado del derecho agrario en el mundo contemporáneo, (29). Recuperado de http://repiica.iica.int/docs/B0075e/B0075e.PDF

Documento recibido el 23 de abril de 2018 y aprobado el 18 de julio de 2018 